\title{
An Evaluation of Post-Incarceration Linkage to Care for HIV/Hepatitis C Infected Veterans in Los Angeles County
}

\section{Henry D Anaya ${ }^{1,2,3, *}$, Jeffery Solomon ${ }^{4}$ and Hemen N Saifu ${ }^{1}$}

${ }^{1}$ Veterans Affairs (VA) Quality Enhancement Research Initiative for HIV and Hepatitis (QUERI-HIV/HEP) and Center for the Study of Healthcare Provider Behavior, VA Greater Los Angeles Health Services Research and Development (HSR and D) Center of Excellence, VA Greater Los Angeles Healthcare System, Los Angeles CA, USA ${ }^{2}$ UCLA David Geffen School of Medicine, Division of General Internal Medicine; Los Angeles, CA, USA

${ }^{3}$ Center for the Management of Complex Chronic Conditions (CM3) and QUERI Spinal Cord Injury (SCI), Chicago, IL, USA

${ }^{4}$ ENRM VA Hospital, Bedford, MA, USA

\begin{abstract}
Objectives: This effort provides the first description of barriers and facilitators that Hepatitis C Virus (HCV) and/or Human Immunodeficiency Virus (HIV) -infected Veterans face in seeking medical care after community re-entry from Los Angeles County (LAC) jails.
\end{abstract}

Design: An evaluation of in-depth qualitative interviews with re-entry HCV and HIV-infected Veterans, and clinical and social service providers. The key variables in the analyses are HIV/HCV linkage to care, barriers/facilitators to linkage to care, and staff perspectives.

Setting: Qualitative data was collected in person at the VA hospital or by telephone interview within the year the study was conducted. Correctional institutions offer opportunities to identify Veterans with HCV and HIV, and for the US Department of Veterans Affairs (VA) to link them to medical care upon release.

Participants: Semi-structured interviews of two cohorts: 1) 16 clinical/non-clinical VA/LAC jail staff; 2) 9 postincarcerated Veterans with HIV and/or HCV.

Main outcome measure: Feedback about barriers and facilitators to reduce gaps, strengthen and improve linkage to care efforts.

Results: Although some characterized efforts positively, barriers were highlighted. Veterans and staff described insufficient strategies for identifying Veterans, ineffective outreach, and inadequate staffing. Strategies for improving linkage, including routine in-take identification of Veteran status, greater program dissemination and increased staff were noted.

Conclusions: Results highlight existing gaps for Veterans transitioning to VA healthcare from LAC jails. Findings can guide future efforts to strengthen gaps between local and federal government.

Keywords: HIV; Hepatitis C; Jail; Veterans

\section{Introduction}

Incarcerated individuals are susceptible to communicable diseases such as Hepatitis-C (HCV) and Human Immunodeficiency Virus (HIV) and have higher rates of HIV and HCV than the general population (HCV: $17 \%$ vs. $1 \%$ ); HIV: $1.6 \%$ vs. $0.5 \%$ ) [1,2]. US Veterans are no exception, and are at higher risk for HIV and HCV than the general population, and have significant incarceration rates, comprising approximately $10 \%$ of all inmates [3]. HIV, once considered a death sentence is now considered a survivable, chronic disease and $\mathrm{HCV}$ is the most common chronic blood borne infection in the US $[4,5]$. The key to managing both conditions is adhering to medication regimens. Lack of adherence leads to numerous complicating factors which may be fatal. However, continuity of medication management is especially challenging for incarcerated Veterans with HIV and/or $\mathrm{HCV}(\mathrm{HIV} / \mathrm{HCV})$. This has much to do with limited access to care within correctional institutions and barriers to obtaining post-release care, e.g., perceived unavailability of civilian health services, [1] lack of health insurance [4] and poor discharge planning [3,5]. Although the healthcare of incarcerated Veterans is the responsibility of correctional facilities, eventually the majority of HCV/HIV-infected Veterans are released from incarceration, where care responsibility shifts to the United States Department of Veterans Affairs Veterans Health Administration (VA) in most instances [6]. It is therefore imperative that robust linkage-to-care mechanisms exist so care is uninterrupted.
Correctional institutions offer an opportunity to identify and treat Veterans with HIV/HCV, and for the VHA to ensure continuity of HIV/ HCV care upon release.

The VA has recognized this, and since 1997, has placed VA outreach specialists (VAOS) in correctional facilities to identify and address the post-release health needs of Veterans through the Health Care for Reentry Veterans (HCRV) Program [6]. The HCRV Program encourages successful community integration, by conducting outreach and facilitating engagement in treatment and rehabilitation. Services include pre-release assessments, referrals to social, psychiatric, and medical services and case management support [6]. This outreach is critical to create a seamless transition for Veterans into the VHA and other supportive community services.

*Corresponding author: Henry D Anaya, UCLA David Geffen School of Medicine Division of General Internal Medicine; Los Angeles, CA, USA, Tel: 310-478-3711, Ext-48488; Fax: 310-268-4933; E-mail: henry.anaya@va.gov

Received June 23, 2014; Accepted August 16, 2015; Published August 25, 2015

Citation: Anaya HD, Solomon J, Saifu HN (2015) An Evaluation of PostIncarceration Linkage to Care for HIV/Hepatitis C Infected Veterans in Los Angeles County. J AIDS Clin Res 6: 493. doi:10.4172/2155-6113.1000493

Copyright: ( 2015 Anaya HD, et al. This is an open-access article distributed under the terms of the Creative Commons Attribution License, which permits unrestricted use, distribution, and reproduction in any medium, provided the original author and source are credited. 
The VA has found that Veterans who had been incarcerated were half as likely as homeless Veterans (38\% vs. 84\%) [7] to utilize any type of VA services upon release from prison. Previously incarcerated Veterans were only slightly less likely to report serious medical problems (33 vs. 37\%) [7]. The gap in access to the VA portends a potential problem with transitional care. Thorough research has been conducted on linking post-incarcerated Veterans who face homelessness, psychiatric issues and substance abuse with VA outreach services when returning to civilian life [7]. However, little is known about the linkage to care patterns that exist for HIV/HCV infected Veterans reentering VA care from jail/prison settings.

The objective of this in-depth qualitative evaluation was to examine the barriers and facilitators that post-incarcerated HIV/HCV infected Veterans encounter when seeking medical care at VA facilities. These interviews present multiple perspectives from infected Veterans and clinical and social service providers. This data is aimed at strengthening the existing linkage to care system by identifying perceptions of VA health services, gaps in care and recommendations to improve and resolve these issues.

\section{Background}

\section{The VA outreach specialist program (VAOS) in the Los Angeles county jail system}

Since 1997, VA Los Angeles has run a Community Re-entry Program that sends VAOS into LAC jails. Staff includes outreach specialists, social workers, and eligibility clerks. The program provides assistance to incarcerated Veterans in preparation for the challenges of community reentry. When an inmate enters the jail system, the Los Angeles County Sheriff's Department (LASD) asks each inmate about his/her Veteran status. A list of self-reported Veterans is provided to program staff that then performs an assessment of eligibility for VA healthcare services. A list of eligible Veterans is then provided to the VAOS. The VAOS perform thorough pre-release assessments to identify the needs of Veterans. They then work with other VA program staff to make necessary referrals and linkages to medical, psychiatric, and social services.

\section{Hepatitis C}

Hepatitis C is well recognized as a public health problem of particular importance to the VA, the largest HCV provider in the US [8]. In 2001, the VA established the National Hepatitis C Program to address all healthcare needs of Veterans with HCV or at risk for HCV including high quality clinical care, prevention efforts, counseling, education, etc. [8]. The prevalence of HCV among Veterans ranges between 5 to $22 \%$ (compared to $1.6 \%$ for non-Veterans) [3,9,10]. A quarter of HCV-infected Veterans may be co-infected with HIV, which greatly increases morbidity from each disease [8]. Access to care for $\mathrm{HCV}$-infected Veterans is critical in order to receive care which is consistent with quality indicators such as assessment of disease severity [11]. Treatment is potentially curative, making outreach programs critical [12].

\section{HIV}

As the largest provider of HIV care in the US, one of the VA's priorities is improving access to HIV care. The VA National HIV Program, initially established as the AIDS Service in 1985, is responsible for advancing policies on clinical care, prevention, testing, education, and research [13]. The prevalence of HIV among Veterans ranges from $0.01 \%$ to $1.85 \%$ when compared to $0.5 \%$ for non-Veterans [14]. With the advent of antiretroviral therapy, HIV has transformed from a fatal to treatable, chronic disease. However, late entry into care, gaps in care, and non-adherence to medication is problematic. This is evident particularly for African-American and Latino men, who constitute the majority of those incarcerated in LAC [15]. In one study, ex-offenders with HIV identified factors that influenced access to primary care and adherence to treatment regimens during their reentry experience. Limited access to transportation, stigma and pessimism about treatment benefits served as obstacles to attending to their HIV needs [15].

HIV and HCV are not only communicable infections but are also chronic infections because of their lifelong complexities. Eighty five percent of those with acute HCV develop chronic HCV and up to $70 \%$ of this group will face liver damage [4]. The virus can last a lifetime, cause cirrhosis or liver cancer and be fatal if left untreated.

Success of antiviral therapy for HIV has dramatically decreased mortality and the risk of AIDS-related illnesses [16]. Although death is not a primary threat for HIV-positive persons with access to treatment, they now face chronic complications such as cardiovascular disease, metabolic issues and end organ damage. It's significant for this population to not only be linked to infectious disease care, but to clinics and providers that are skilled in caring for their comorbidities.

Having chronic conditions cause people to adjust their lives to meet their healthcare needs. When someone with $\mathrm{HIV} / \mathrm{HCV}$ is managing their disease it is difficult to continue their treatment regimen when care continuity is disrupted by arrest and incarceration. Research suggests that prisoners do not receive the standard of care they were receiving prior to incarceration, which can increase their risk of complications or poor outcomes [17]. It's important for this population to be linked to care immediately post-release so they can continue their established plan of care.

\section{Methods}

A VA IRB board reviewed and sanctioned all issues and methods regarding interviews with Veterans and with staff. Verbal informed consent was obtained prior to each interview.

This study was a formative qualitative evaluation that entailed semistructured interviews, conducted by one of the investigators (HS), with two separate cohorts of participants: Formerly-incarcerated Veterans, who were HIV and/or Hepatitis-C positive, and clinical and nonclinical VA and LAC jail staff.

Veterans were eligible for participation if they had been contacted by a VAOS at one of the Los Angeles Sheriff's Department (LASD) jail facilities, were released from jail within the past year, and self-reported a history of either HIV and/or Hepatitis-C infection. In addition, they had to be honorably discharged from military service and eligible for VA services.

VAOS assisted with recruiting Veterans by identifying those with self-reported HIV/HCV and distributing informational project fliers in the jails. Fliers indicated that Veterans could seek to enroll in the study either by contacting one of the investigators or providing their contact information to the VAOS, who would then forward it to the research team.

Interviews with Veterans were designed to elicit their perceptions of, and experiences with the linkage to care process. A total of nine Veterans were interviewed, 8 with HCV and 1 with HIV. Interview questions asked Veterans to describe how they learned about linkage to care issues, experiences transitioning from the jail system to VA 
care (barriers and facilitators), their perceptions of linkage to care personnel, and their perceptions of VA care. Interviews were conducted in English, lasted approximately 20-30 minutes, and took place in an investigator's office. Financial incentives in the form of a 50 dollar VA canteen ${ }^{1}$ voucher were provided upon completion (VA IRB determined that this incentive was not coercive in nature).

Clinical and non-clinical VA and LAC jail staff was identified as potential participants by the principal investigator (PI). The project manager sent emails to 25 staff providing a project outline and inviting them to participate in a semi-structured interview. Interviews were conducted in English by the project manager, either by telephone or in-person, depending on participants' preferences. Upon completion of interviews, the project manager used snowball sampling to identify additional potential participants. There were no incentives offered for this cohort.

A total of 16 clinical and non-clinical VA and LAC jail staff was interviewed. Interviews lasted approximately 25 minutes and addressed the following topics: The current state of linkage to any post-release care and to VA post-release care (both general and disease-specific), the perceived barriers and facilitators to Veterans' accessing care, Veterans' knowledge and awareness of their diseases and VA resources for treatment, identifying methods to improve linkage to care efforts and challenges in providing care to this population. No demographic information was recorded for either Veterans or staff.

Interviews with all participants in both cohorts were audiorecorded, transcribed verbatim, and uploaded onto a secure VA network. Transcripts were uploaded to a qualitative coding software program (NVivo 10) and analyzed by the qualitative lead (JS). A thematic analysis approach was used, which entailed utilizing both $a$ priori and inductive frameworks for coding data according to thematic categories and, when warranted, sub-categories. Throughout the analysis, ongoing comparisons were made within both Veteran and staff cohorts and, eventually, between cohorts. Once an initial coding and data interpretation were complete, preliminary findings were shared with the full study team, who provided feedback and key insights. This feedback was integrated into revised findings and discussed again among the team. The team achieved consensus regarding the revised findings.

\section{Results}

\section{Veterans' perspectives}

Facilitators for creating linkages to HCRV: The key facilitator described by Veterans for creating linkages to HCRV was being contacted by the VAOS, or a network set up by VAOS, during incarceration. As a Veteran explained,

"As soon as I was incarcerated a red flag went off on the computers with the sheriff's department and the VA and VA representatives. I didn't have to put in a request for them to come see me. They were already notified I was in jail."

As implied in this example, it is especially beneficial when eligible Veterans are identified upon entering jail, rather than the responsibility falling on Veterans to request contact with a VAOS.

However, establishing contact with a VAOS was possible when Veterans were not flagged by the computer system. For example, a

1 VA canteens are small retail stores that are onsite in larger VA hospitals; snack food and other sundry items are available for patients, family members and VA employees.
Veteran described first meeting with a social worker, who then arranged for the VAOS to contact him:

"I was speaking with a social worker, and she asked if I was a Veteran. And I said 'yes'. She then asked if they can contact the VA. I said, 'Certainly'. Next thing I know, a man comes up to me in the [X] jail, named $[\mathrm{X}]$ from the VA, and [he] interviewed me."

This example indicates that at least some social work staff is aware of the VAOS service and also sufficiently prioritizes Veteran linkage to care to inquire about inmates' Veteran status.

Barriers to creating linkages to HCRV: Notably, some Veterans depicted the opposite of the facilitators noted above, as key barriers to developing linkages to HCRV. Specifically, barriers centered on a perceived lack of jail/VAOS linkage and a lack of disseminated information about HCRV. For example, one Veteran noted that if he had not discovered a HCRV-related pamphlet on the floor by happenstance, he would not have known about the program. Another said he had requested to meet with VA representatives while incarcerated, but that never happened.

Veterans' views on improving linkages to HCRV: Many of the Veterans who described barriers to accessing HCRV went on to make suggestions for improving HCRV linkage. Some Veterans suggested developing more effective outreach communication strategies, including posting flyers or other signage in jail cells and other jail locations. For example, one Veteran commented:

“There should be some kind of sign that says, 'Attention Veterans', just like I saw in the jail dorms in Florida....Post something that lets Vets know, 'We'll be out here on such and such a date. If you have any questions about health concerns, matters, or services, come to this meeting.' This should be posted on the bulletin boards."

This example suggests that Veterans might take the initiative to contact appropriate personnel if information about services were visible.

In a different vein, another Veteran expressed that more VAOS's are needed in the jails to address Veterans' needs. As he explained:

"There should be more liaisons in the jail...I only see a couple, but there's not enough coming in there. And they should make it a priority for the VA reps to work with the sheriff's department to make sure that all Veterans are identified, period. All Veterans should be seen by a VA rep, most of the time you have to request this. But a lot of guys don't even know about this."

One Veteran appears to contradict what another had identified as a facilitator (see above) by stating that there should be a system in place to notify VAOS's of newly incarcerated Veterans. As he explained:

"As long as anyone's a vet, and that should be something the sheriff's department asks this, and that red flag goes up when you're incarcerated. And you're a vet so that the VA representatives know you're there. To know someone's there for you while you're incarcerated helps and makes time go by easier."

Once linked to VA care, Veterans mostly described positive perceptions of it. They characterized care in general to be "good" and even patient-centered. More specific positive views pertained to the quality of HCV and HIV treatment and the availability of care for those with low or no income.

There were some negative views of VA care, such as having to wait long periods until scheduled appointments and the challenges of 
filling prescriptions. Three Veterans also noted they were not aware of important VA resources to manage their Hepatitis $\mathrm{C}$ like the Hepatitis clinic and Hepatitis C education classes.

"I've just been working through my primary care doctors at the domiciliary; they have good doctors and nurses there. They've never told me about the Hepatitis $\mathrm{C}$ clinic; I'm going to mention it to my primary care provider. Where is this Hep C Clinic located? I'd like to go to that clinic."

"I didn't know about the Hepatitis C Clinic education class. You're helping me now!"

At the end of these interviews, the interviewer instructed Veterans on how to access the education class and the location of the Hepatitis clinic.

\section{Cohort 2: LAC/VA staff perspectives}

Facilitators for creating linkages to HCRV: The first facilitator identified by staff had a system that effectively identified potentially eligible Veterans. As a VAOS commented,

“...this system can't be any more efficient. I mean right when they [Veterans] get to jail they're identified unless they say no to the sheriff."

Notably, this perspective accords with some Veterans' views, on the one hand, and contradicts others' views, on the other. For example, we earlier described a Veteran's perception that Veteran status is automatically "flagged" in a computer system upon entering the jail. However, we also referenced another Veteran's perception that there is a need for a system for identifying eligible Veterans when they arrive at the jail. A second facilitator mentioned by staff for generating linkages to HCRV was fostering positive relationships with Veterans, and even with fellow staff members. For instance, a VA case manager said:

"I try to always make a good impression when first meeting them [Veterans] and let them know what I'm there for, and things that I can help them with....Each time I meet with them, I think they develop more of a sense of trust."

This example suggests that merely providing Veterans with information about the program's existence is inadequate. Indeed, as the case manager notes, both making "a good impression" and cultivating trust are key qualities necessary for creating potential linkages to HCRV.

Another VA case manager described the importance of having good working relationships with colleagues, noting:

"What's very helpful is having that great rapport, because it takes a team....so just having that relationship, the good rapport, as a team, benefits the Veterans."

This example highlights the fact that transitioning Veterans to HCRV requires more than the efforts of a single person. Specifically, it points out that such transitions are the coordinated efforts of multiple staff, both at the jail and the VA.

\section{Barriers to creating linkages to HCRV}

Some staff echoed the same barriers to HCRV described by Veterans. For example, a VA case manager spoke to the challenge of staffing insufficiencies in the jail when commenting:

"I would assume that the outreach program is out-gunned, undermanned...there's only so much penetration [in jails] they can do."

Other staff, however, described barriers pertaining to the VA itself rather than linkages to HCRV per se. For example, a VA case manager said:

"An overarching problem is that our volume is so high and there are long waits to be seen by staff and lack of beds to house Veterans, which is an issue of access to care."

This example suggests that even if linkages between the jail and HCRV were optimal, Veterans would still experience access problems due to VA staff and bed shortages.

Finally, staff depicted various patient factors that are barriers to their success once linked to VA care. Such factors included mental illness, substance abuse, resistance to taking instruction from staff, loss of--and non-adherence to--medications, stigmatization (both as it related to incarceration and their infection status), negative preconceptions of the VA, missing appointments, and unfamiliarity with the medical system. As an example of the role stigmatization plays, a VA case manager said, "I think sometimes the Veterans feel stigmatized and not as open to talk about these issues."

Staff also indicated that in some cases Veterans who are released from jail state they will enter VA care but in fact do not attend. As a provider explained:

"The barriers are that people get out of jail and get caught up in the same old stuff. They may tell us they're going to [name of VA treatment facility] to get treatment of substance abuse or mental health issues, but they never make it, and they may end up downtown and start using [drugs] again."

Staff views on improving linkages to HCRV: When it comes to suggested improvements to HCRV, staff echoed many views articulated by Veterans. Suggested improvements included arranging for both VA and non-VA staff to learn more about resources for Veterans, dedicating more staff to HCRV, developing better strategies for identifying incarcerated Veterans, and using more effective means of informing Veterans about HCRV, (e.g., flyers posted in jails).

Case managers for both VA and non-VA suggested training health care staff on how to talk more effectively with Veterans. VA case managers suggested conducting outreach at venues attended by Veterans such as motocross races. Non-VA case managers recommended creating faster linkages to care once Veterans are released and establishing a HCRV telephone hotline. Specialty providers recommended the ability to access Veterans' prison records and discharge plans via the VA Computerized Patient Record System (CPRS), and improving communication between outreach specialists and themselves.

\section{Discussion}

This effort was initiated to gather foundational evidence regarding management of care, care coordination and continuity of care for HIV/ HCV-infected Veterans transitioning to VA care from the Los Angeles County jail system. As expected with a divergent interview sample, perceptions regarding what works and what don't, at the individual and organizational level can be complex enough to create ambiguity when drawing specific conclusions and possible improvement recommendations. With this said, some issues seem simple enough to flesh out for further investigation and possible improvement. For example, there was mention of the lack of awareness by some Veterans regarding integration with the HCRV program. This can be easily remedied with an increased effort to highlight and promote the benefits available to transitioning Veterans, and specifically, Veterans infected with $\mathrm{HIV} / \mathrm{HCV}$. The posting of flyers, brochures, etc., within common areas of the jails are some suggestions made by Veterans. 
Citation: Anaya HD, Solomon J, Saifu HN (2015) An Evaluation of Post-Incarceration Linkage to Care for HIV/Hepatitis C Infected Veterans in Los Angeles County. J AIDS Clin Res 6: 493. doi:10.4172/2155-6113.1000493

Page 5 of 5

Some other suggestions, such as increasing the number of VAOS available in the jail system, a sentiment expressed by both Veterans and staff, will be difficult to pursue. However, it further highlights the perceptions among some Veterans that more could be done to increase the awareness of what programs and services are available to incarcerated Veterans reintegrating into the community and transitioning to the VA for their healthcare.

\section{Limitations}

The potential for coercion in using an incentive as a data gathering technique regarding our Veteran interviews was discussed prior to the start of the project; we did not feel our incentive structure undermined or negatively influenced participant response. In addition, our local IRB board did not deem this to be an overly coercive influence; however, there is always the potential for incentives to be deemed coercive.

Due to the brief 1 year project timeline and specific population criteria for interviews, our local IRB limited our interviewee sample size to a maximum of 9 Veteran interviews. This study initially aimed to interview approximately 5 Veterans with HCV and 4 with HIV, however our VAOS had difficulty finding HIV-infected Veterans for this study, hence the imbalance ( 8 to 1 ) in this measure. Also, since we were evaluating a vulnerable population, our IRB did not allow the collection of demographic data. Nor did the IRB allow job classification for staff. Also, because the focus of this project targeted Veterans, this may restrict the applicability of this analysis to the wider healthcare system.

\section{Conclusions}

These findings can contribute to interventions to make HCRV a stronger, more effective program, with the hope that more recently released Veterans will be linked to HIV and/or HCV care. It's significant for this population to have continuous care as they attempt to manage all of their complications from these lifelong chronic viruses. Because these findings underscore a gap in processes between the LAC jail system and the VA in regard to knowledge of linkage to care issues for HIV and/or Hepatitis-C infected Veterans, future interventions to address these gaps would be beneficial to both Veterans and the VA healthcare system. Such interventions would be part of the VA's contribution to the federal government's response to the National HIV/ AIDS strategy, which specifically calls for increasing access to care and outreach efforts, especially efforts to increase linkage to care.

Finally, future interventions, paired with ongoing research evaluations could have wide-ranging effects on best practices approaches regarding implementation-based research efforts. Current plans are to use the results of this formative needs assessment as the basis of future interventions aimed at strengthening the currently existing linkage-tocare networks between local and federal systems.

\section{Acknowledgements}

The authors would like to thank Dr. Sonali Kulkarni of the Los Angeles County Department of Health and the Los Angeles VAOS staff for their assistance with this effort.

This research was funded by VA Quality Enhancement Research Initiative (QUERI) Rapid Response Project (RRP) grant RRP 10-182 awarded to the first author and supported by the Department of Veterans Affairs, Veterans Health Administration (VHA), Health Services Research and Development Service (HSR and D).

\section{References}

1. Wilper AP, Woolhandler S, Boyd JW, Lasser KE, McCormick D, et al. (2009) The health and health care of US prisoners: Results of a nationwide survey. Am J Public Health 99: 666-672.

2. Rich JD, Allen SA, Williams BA (2014) Responding to Hepatitis C through the Criminal Justice System. N Engl J Med 370: 1871-1874.

3. Dominitz JA, Boyko EJ, Koepsell TD, Heagerty PJ, Maynard C, et al. (2005) Elevated prevalence of hepatitis $C$ infection in users of United States veterans medical centers. Hepatology 41: 88-96.

4. Hepatitis C FAQs for the Public. Division of Viral Hepatitis, Centers for Disease Control and Prevention.

5. Goetz MB, Hoang T, Bowman C, Knapp H, Rossman B, et al. (2008) A systemwide intervention to improve HIV testing in the Veterans Health Administration. J Gen Intern Med 23: 1200-1207.

6. VHA Handbook 1162.06. (2010) Health Care for Re-Entry Veterans (HCRV) Program.

7. McGuire J, Rosenheck RA, Kasprow WJ (2003) Health status, service use, and costs among veterans receiving outreach services in jail or community settings. Psychiatr Serv 54: 201-207.

8. VHA Directive 2007-022 (2013) National Viral Hepatitis C Program.

9. Roselle GA, Danko LH, Kralovic SM, Simbartl LA, Kizer KW (2002) National Hepatitis $C$ surveillance day in the Veterans Health Administration of the Department of Veterans Affairs. Military Medicine 167: 756-759.

10. Sloan KL, Straits-Tröster KA, Dominitz JA, Kivlahan DR (2004) Hepatitis C tested prevalence and comorbidities among veterans in the US Northwest. J Clin Gastroenterol 38: 279-284.

11. Physician Quality Reporting System (2010) Centers for Medicare and Medicaid services.

12. McHutchison JG, Manns MP, Muir AJ, Terrault NA, Jacobson IM, et al. (2010) Telaprevir for previously treated chronic HCV infection. N Engl J Med 362: 1292-1303.

13. VHA Directive 1304 (2014) NATIONAL HUMAN IMMUNODEFICIENCY VIRUS (HIV) PROGRAM. Department of Veterans Affairs VHA Directive 1304, Veterans Health Administration Transmittal Sheet.

14. Owens DK, Sundaram V, Lazzeroni LC, Douglass LR, Sanders GD, et al. (2007) Prevalence of HIV Infection Among Inpatients and Outpatients in Department of Veterans Affairs Health Care Systems: Implications for Screening Programs for HIV. Am J Public Health 97: 2173-2178.

15. Fontana L, Beckerman A (2007) Recently released with HIVIAIDS: primary care treatment needs and experiences. J Health Care Poor Underserved 18: 699714

16. Deeks SG, Lewin SR, Havlir DV (2013) The end of AIDS: HIV infection as a chronic disease. Lancet 382: 1525-1533.

17. AIDS InfoNet (2014) HIV in Prisons and Jails. New Mexico AIDS Education and Training Center at the University of New Mexico Health Sciences Center, and the International Association of Providers of AIDS Care. 